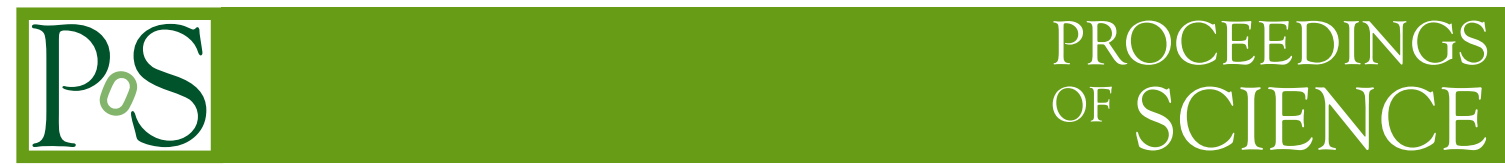

\title{
Charged Higgs bosons at the Compact Linear Collider
}

\author{
Elias Coniavitis* \\ Uppsala University \\ E-mail: elias.coniavitis@physics.uu.se \\ Arnaud Ferrari \\ Uppsala University \\ E-mail: arnaud.ferrari@physics.uu.se
}

The pair production of charged Higgs bosons in $e^{+} e^{-}$collisions at a centre-of-mass energy of $3 \mathrm{TeV}$, e.g. at the proposed Compact Linear Collider (CLIC), could allow precise measurement of the $H^{ \pm}$mass and of $\tan \beta$. A study of this process has been performed, using a parametrized generic linear collider detector simulation and taking into account high-energy beam-beam effects at the interaction point.

Prospects for Charged Higgs Discovery at Colliders

16-19 September 2008

Uppsala, Sweden

\footnotetext{
* Speaker.
} 


\section{Introduction}

The exploration of the Higgs sector will be a major task for any future high energy collider. While not part of the Standard Model (SM) Higgs sector, charged Higgs bosons can be crucial for exploring an extended Higgs sector, such as the Two Higgs Doublet Model of the Minimal Supersymmetric extension of the SM (MSSM), should that be realized in nature.

The Large Hadron Collider (LHC) has the potential to probe a substantial part of the parameter space of an extended Higgs sector. However, discovering $H^{ \pm}$bosons can be very challenging, in particular if $H^{ \pm}$is heavy [1]. At the same time, it will also be difficult to achieve precise determination of important parameters, such as $\tan \beta$ (the ratio of the two Higgs doublets' vacuum expectation values) and the charged Higgs boson mass.

These challenges could be tackled at a future multi-TeV linear collider, such as the proposed $e^{+} e^{-}$Compact Linear Collider [组] (CLIC), operating at a nominal centre-of-mass energy of $3 \mathrm{TeV}$. The more precise knowledge of the collision energy, the cleaner environment and the fewer backgrounds compared with the LHC should enable a more detailed exploration of the Higgs sector.

In this study, the production of $\mathrm{H}^{+} \mathrm{H}^{-}$pairs at CLIC has been investigated in the framwork of the MSSM, with a focus on the mass range from 0.5 to $1.5 \mathrm{TeV}$. Two channels were considered, $e^{+} e^{-} \rightarrow H^{+} H^{-} \rightarrow t b t b$ and $e^{+} e^{-} \rightarrow H^{+} H^{-} \rightarrow t b \tau v^{1}$. The $H^{ \pm}$decay width and branching ratios were calculated using HDECAY [3], PYTHIA 6.342 [4] was used to generate the signal samples and MadGraph/MadEvent [5] the backgrounds, while SIMDET [6] was used to perform a parametrized detector simulation. Supersymmetric decays of the $H^{ \pm}$as well as supersymmetric backgrounds were not considered.

\section{Beam-beam effects}

At CLIC the incoming beams will lose on average $16 \%$ of their energy following the emission of high-energy photons due to beam-beam effects (beamstrahlung). This means that even though the nominal incoming beam energy is $1.5 \mathrm{TeV}$, the actual centre-of-mass energy will have long tails below $3 \mathrm{TeV}$ as shown in figure 1(a). The emitted high-energy photons may then collide and produce a hadronic background (on average 0.73 events per bunch crossing). All these effects have been taken into account. Figure 1 (b) shows the effect of the modified centre-of-mass energy due to beam-beam effects on the production cross-section for $\mathrm{H}^{+} \mathrm{H}^{-}$pairs.

\section{Analysis}

An outline of the analysis performed on the two studied channels is given below. For a more detailed discussion, see [7].

\section{$3.1 e^{+} e^{-} \rightarrow H^{+} H^{-} \rightarrow t b t b$}

In a first stage, cuts are performed to only keep those events that match the signal final state, where only the hadronic decays of the $W$ boson are considered, i.e. one demands the presence

\footnotetext{
${ }^{1}$ This notation will be used in the following to indicate both of the charge conjugate processes involved in each case, i.e. $e^{+} e^{-} \rightarrow H^{+} H^{-} \rightarrow t b \tau \nu$ stands for $e^{+} e^{-} \rightarrow H^{+} H^{-} \rightarrow t \bar{b} \tau \bar{v}$ and $e^{+} e^{-} \rightarrow H^{+} H^{-} \rightarrow \bar{t} b \bar{\tau} v$
} 


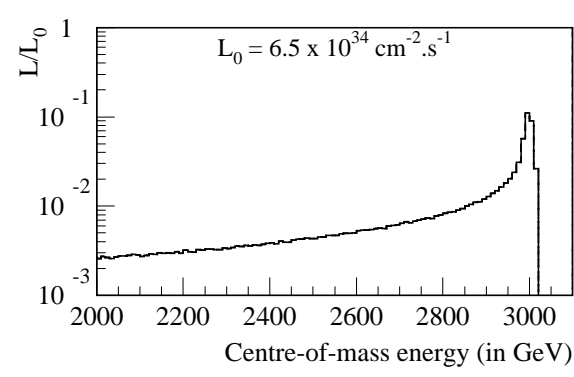

(a)

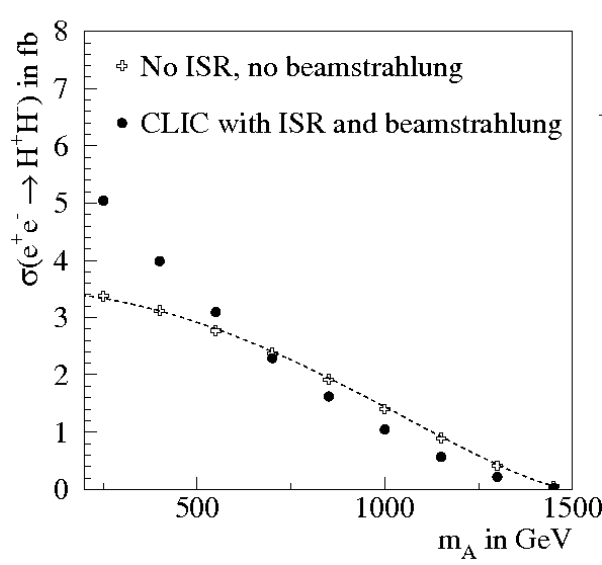

(b)

Figure 1: (a): Centre-of-mass energy spectrum at CLIC, after the inclusion of beam-beam effects. (b): Production cross section for $\mathrm{H}^{+} \mathrm{H}^{-}$pairs at CLIC. The open crosses and the dashed line show the cross sections calculated at tree level, with PYTHIA and analytically, respectively, while the full circles show the cross sections obtained after taking the beam-beam effects into account.

of at least eight jets, exactly four of them b-tagged, and no isolated electron or muon. The two $W$ bosons and $t$ quarks are then reconstructed, by taking the two combinations which minimize $\delta=\left|m(a, b)-m_{W / t}\right|$ (where $a, b$ are non-b-jets in the $W$ case and a b-jet and a $W$ candidate in the top case). Cuts on the reconstructed masses requiring $50<m_{W r e c}<150 \mathrm{GeV}$ as well as $100<$ $m_{\text {trec }}<300 \mathrm{GeV}$ are applied. For the reconstruction of the charged Higgs boson, each top quark candidate is assigned a b-jet such that $\Delta_{t b}=\left|m(t b)_{1}-m(t b)_{2}\right|$ is minimized, since the two charged Higgs candidates should have the same mass. If $\Delta_{t b}>250 \mathrm{GeV}$ the event is discarded. Two additional cuts are performed to reduce the SM-like backgrounds: $m_{r e c}(t t)-m_{\text {rec }}(b b)<1 \mathrm{TeV}$, and $\left[m_{\text {rec }}(t t)+m_{\text {rec }}(b b)\right]>2 \mathrm{TeV}$.

A kinematical fit is applied to further improve the reconstruction. If an event fails the kinematical fit, it is discarded. Figure $2\left(\right.$ a) shows the reconstructed mass spectrum of the $H^{+} H^{-}$pairs for an integrated luminosity of $3000 \mathrm{fb}^{-1}$, as obtained with $m_{A}=700 \mathrm{GeV}$ and $\tan \beta=25$, before and after the kinematical fit is applied. An improvement of the mass resolution by $50 \%$ is clearly visible. Table 1 displays the expected number of signal and background events for $m_{A}=1 \mathrm{TeV}$, in $\mathrm{a} \pm 2.5 \sigma$ window around the charged Higgs boson mass peak.

\section{$3.2 e^{+} e^{-} \rightarrow H^{+} H^{-} \rightarrow t b \tau \nu$}

As in the previous channel, the first step is to demand a final state matching that of the signal (where only hadronic $W$ and $\tau$ decays are considered). Hence, events are required to have no isolated lepton, at least $400 \mathrm{GeV}$ missing energy and at least five jets, of which exactly two are b-tagged and exactly one $\tau$-tagged. The reconstruction of the charged Higgs boson that decayed into quarks proceeds as described in the previous section, while for the $H^{ \pm}$that decayed to $\tau \nu$ only the transverse mass can be reconstructed due to the presence of neutrinos (shown in figure 2(b)). This transverse mass is required to be greater than $150 \mathrm{GeV}$. To reduce the SM-like backgrounds, 


\begin{tabular}{ccccc} 
Process & Efficiency & \multicolumn{3}{c}{$N_{\text {events }}$ when $\tan \beta$ is } \\
of interest & $\varepsilon$ (in \%) & small & intermediate (7.6) & large \\
\hline$H^{+} H^{-} \rightarrow t b t b$ & 1.56 & 49.0 & 42.0 & 36.7 \\
\hline$e^{+} e^{-} \rightarrow t b t b(\mathrm{SM})$ & 0.03 & 1.8 & 1.8 & 1.8 \\
$e^{+} e^{-} \rightarrow b b b b(\mathrm{SM})$ & 0.02 & 1.6 & 1.6 & 1.6 \\
$e^{+} e^{-} \rightarrow t t t t(\mathrm{SM})$ & 0.05 & - & - & -
\end{tabular}

Table 1: Expected number of signal and background events for $e^{+} e^{-} \rightarrow H^{+} H^{-} \rightarrow t b t b$ with $m_{A}=1 \mathrm{TeV}$ and an integrated luminosity of $3000 \mathrm{fb}^{-1}$.

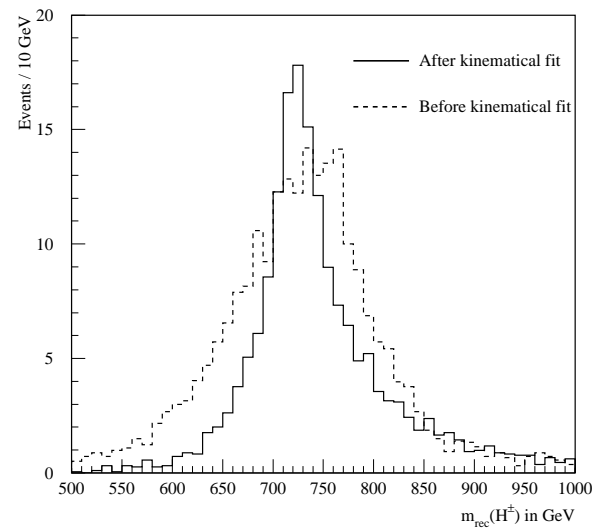

(a)

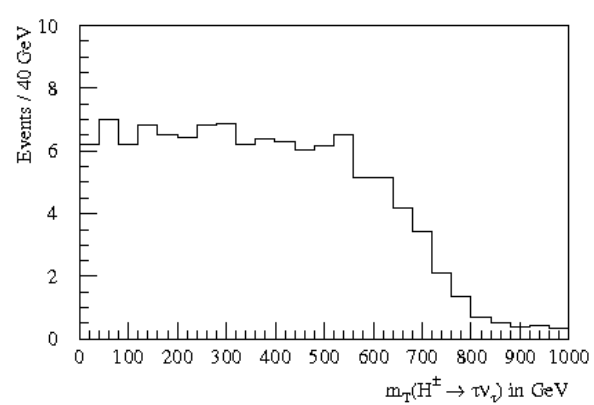

(b)

Figure 2: (a): Mass reconstruction of $H^{+} H^{-}$pairs from the $t b t b$ final state, before (dashed line) and after (full line) use of the mass constrained kinematical fit. (b): Transverse mass of $H^{+} \rightarrow \tau v$. Both plots are for an integrated luminosity of $3000 \mathrm{fb}^{-1}$, with $m_{A}=700 \mathrm{GeV}$ and $\tan \beta=25$.

the angle of the two $H^{ \pm}$candidates in the transverse plane is required to be greater than $2.4 \mathrm{rad}$ (since they should be back-to-back, as opposed to most backgrounds). Table 2 shows the expected amount of signal and background events for $m_{A}=1 \mathrm{TeV}$, in a $\pm 2.5 \sigma$ window around the charged Higgs boson mass peak (in the $m_{\text {rec }}(t b)$ spectrum), after all selection cuts.

\begin{tabular}{ccc}
$\begin{array}{c}\text { Process } \\
\text { of interest }\end{array}$ & $\begin{array}{c}\text { Efficiency } \\
\varepsilon \text { (in \%) }\end{array}$ & $\begin{array}{c}N_{\text {events }} \\
\text { at large } \tan \beta\end{array}$ \\
\hline$H^{+} H^{-} \rightarrow t b \tau \nu_{\tau}$ & 4.55 & 32.9 \\
\hline$e^{+} e^{-} \rightarrow t b \tau \nu_{\tau}(\mathrm{SM})$ & 0.02 & 5.0 \\
$e^{+} e^{-} \rightarrow b b \tau \tau(\mathrm{SM})$ & 0.01 & 0.1 \\
$e^{+} e^{-} \rightarrow t t \tau \tau(\mathrm{SM})$ & 0.05 & 0.1
\end{tabular}

Table 2: Expected number of signal and background events for $e^{+} e^{-} \rightarrow H^{+} H^{-} \rightarrow t b \tau v_{\tau}$ with $m_{A}=1 \mathrm{TeV}$ and an integrated luminosity of $3000 \mathrm{fb}^{-1}$, at large $\tan \beta$. 


\subsection{Discovery Contour}

For a discovery, the signal $S$ is required to be at least 10 events and, in addition, it must exceed 5 statistical fluctuations of the background $B$, i.e. $S / \sqrt{B} \geq 5$. Systematic uncertainties have not been considered. The discovery contour can be seen in figure B: CLIC has the potential to reach masses well above $1 \mathrm{TeV}$ regardless of $\tan \beta$.

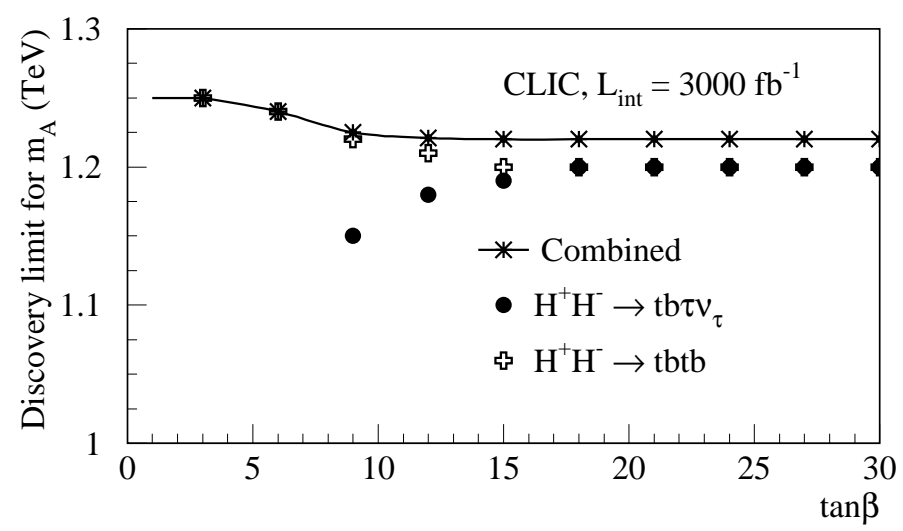

Figure 3: Discovery contour in the $\left(\tan \beta, m_{A}\right)$ plane for $H^{+} H^{-}$pairs at CLIC, for an integrated luminosity of $3000 \mathrm{fb}^{-1}$.

\section{Parameter Determination}

Discovering a charged Higgs boson is of course an extremely important step in exploring an extended Higgs sector, however further exploration will require the precise determination of $\tan \beta$ and the mass of one of the Higgs bosons (conventionally $m_{A}$ is used) - which are the two parameters which fully describe the Higgs sector of the MSSM at tree level.

\subsection{Higgs mass parameter $m_{A}$}

In order to determine the expected statistical accuracy achievable at CLIC for measuring the $m_{A}$ variable (on which the $H^{ \pm}$mass depends, through the relation $m_{H^{ \pm}}^{2}=m_{A}^{2}+m_{W}^{2}$ ) a $\chi^{2}$-fit is performed: a sample of "real" $e^{+} e^{-} \rightarrow H^{+} H^{-} \rightarrow t b t b$ events were generated, along with the corresponding "real" SM backgrounds. Thereafter "simulated" samples (signal and background) of very high statistics were generated over a span of different values of $m_{A}$. Each of these samples can then be normalized to a number of different cross-sections. For each normalized sample of "simulated" events, the $\chi^{2}$ is calculated as:

$$
\chi^{2}=\sum_{i} \frac{\left(N_{r}(i)-N_{s}(i)\right)^{2}}{N_{r}(i)}
$$

with $N_{s}(i)$ and $N_{r}(i)$ being the number of "simulated" and "real" events in the $i$ :th bin of the charged Higgs boson mass histogram. For each value of $m_{A}$ the cross-section normalization that yields the 
minimal $\chi^{2}$ is thus obtained. Using a parabolic fit one can then obtain the $m_{A}$ value corresponding to the lowest $\chi^{2}$ value - along with the statistical uncertainty.

Applying this method to a "real" sample with $m_{A}=700 \mathrm{GeV}$, for two different $\tan \beta$ ranges, leads to the results shown in table 3 . The statistical uncertainty remains below $1 \%$.

\begin{tabular}{lcc} 
Configuration & $m_{A}(\mathrm{GeV})$ & $\delta m_{A}(\mathrm{GeV})$ \\
\hline Small $\tan \beta$ & 701.2 & 3.7 \\
Large $\tan \beta$ & 701.8 & 4.9
\end{tabular}

Table 3: Reconstructed value of $m_{A}$, with its statistical error, derived from a $\chi^{2}$-analysis of $e^{+} e^{-} \rightarrow$ $H^{+} H^{-} \rightarrow t b t b$ events, with $m_{A}=700 \mathrm{GeV}$ and an integrated luminosity of $3000 \mathrm{fb}^{-1}$.

\section{$4.2 \tan \beta$}

The ratio of the partial decay widths of the two studied processes depends on the variable $\tan \beta$ at tree level according to:

$$
\frac{\Gamma\left(H^{ \pm} \rightarrow t b\right)}{\Gamma\left(H^{ \pm} \rightarrow \tau v_{\tau}\right)}=\frac{3}{m_{\tau}^{2}}\left[\bar{m}_{t}^{2} \cot ^{4} \beta+\bar{m}_{b}^{2}\right]
$$

with $\bar{m}_{q}$ standing for the running mass of the quark $q$. This $\tan \beta$ dependence is demonstrated in the left-hand side plot of figure 4 .

The variable $\tan \beta$ can thus be determined experimentally through the ratio $R^{ \pm}$of the signal rates of the two studied processes ( $\varepsilon$ being the signal selection efficiency, which does not depend on $\tan \beta)$ :

$$
R^{ \pm}=\frac{N_{t b t b}}{N_{t b \tau v_{\tau}}}=\frac{\Gamma\left(H^{ \pm} \rightarrow t b\right)}{\Gamma\left(H^{ \pm} \rightarrow \tau v_{\tau}\right)} \times \frac{\varepsilon_{t b t b}}{2 \varepsilon_{t b \tau v_{\tau}}} .
$$

The statistical uncertainty on $R^{ \pm}$is:

$$
\frac{\delta R^{ \pm}}{R^{ \pm}}=\sqrt{\left(\frac{\delta(\sigma \times \mathrm{Br})}{\sigma \times \mathrm{Br}}\right)_{t b t b}^{2}+\left(\frac{\delta(\sigma \times \mathrm{Br})}{\sigma \times \mathrm{Br}}\right)_{t b \tau v_{\tau}}^{2}} .
$$

The right-hand side plot of figure 4 shows how the expected statistical error on $\tan \beta$ depends on this parameter. At too small $\tan \beta, e^{+} e^{-} \rightarrow H^{+} H^{-} \rightarrow t b \tau \nu$ has a vanishing branching ratio, while for $\tan \beta$ larger than about 12 the ratio $R^{ \pm}$has an asymptotic behaviour, leading to a relative error that is too large for a precise measurement. The best determination of $\tan \beta$ is thus achieved in the intermediate region, around $\tan \beta=7$.

\section{Conclusions}

CLIC has the potential to cover precisely the regions of the charged Higgs sector which are the most challenging at the LHC. It has a discovery potential for the charged Higgs boson reaching up to masses beyond $1 \mathrm{TeV}$ regardless of $\tan \beta$, while it could also allow for the precise determination of the parameters describing the Higgs sector of the MSSM at tree level: $m_{A}$ can be determined with a statistical uncertainty of about $1 \%$, while $\tan \beta$ can be determined with a statistical uncertainty of 

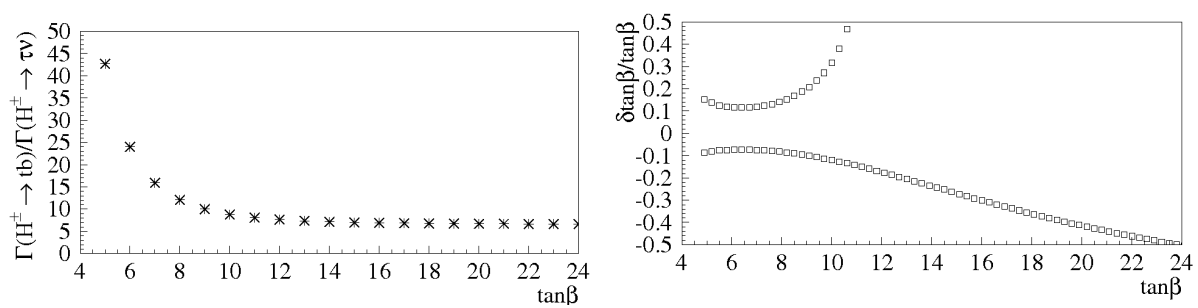

Figure 4: Variation with $\tan \beta$ of the ratio between the two partial decay widths of the charged Higgs boson (left) and of the relative error on $\tan \beta$ when deriving this parameter from the ratio of the signal rates for $H^{+} H^{-} \rightarrow t b t b$ and $H^{+} H^{-} \rightarrow t b \tau \nu_{\tau}$ (right), for $m_{A}=700 \mathrm{GeV}$ and an integrated luminosity of $3000 \mathrm{fb}^{-1}$.

down to about $10 \%$, if it has values around 7. CLIC could thus be an excellent tool for probing an extended Higgs sector.

This study necessarily remains preliminary, since both the CLIC machine and its detector are still at an early, conceptual design phase. Once a final design has been settled upon, this study would have to be repeated using a full and more realistic detector simulation, taking systematic uncertainties into account, and potentially also with the knowledge of new physics results achieved at the LHC.

\section{References}

[1] M. Flechl Charged Higgs Prospects with ATLAS, this volume. R. Kinnunen Charged Higgs Prospects with CMS, this volume.

[2] The CLIC Study Team, CERN 2000-008.

[3] A. Djouadi, J. Kalinowski and M. Spira, Comp. Phys. Comm. 10856 (1998).

[4] T. Sjöstrand, P. Eden, C. Friberg, L. Lonnblad, G. Miu, S. Mrenna and E. Norrbin, Comp. Phys. Comm. 135238 (2001).

[5] F. Maltoni and T. Stelzer, Preprint hep-ph/0208156; W.F. Long and T. Stelzer, Comp. Phys. Comm. 81 357 (1994); K. Hagiwara, H. Murayama and I. Watanabe, Preprint KEK Report 91-11.

[6] M. Pohl and H.J. Schreiber, Preprint DESY 99-030.

[7] E. Coniavitis and A. Ferrari, Phys. Rev. D75 (2007) 015004 